“ (C) 2019 IEEE. Personal use of this material is permitted. Permission from IEEE must be obtained for all other uses, in any current or future media, including

reprinting/republishing this material for advertising or promotional purposes, creating new collective works, for resale or redistribution to servers or lists, or reuse of any copyrighted component of this work in other works." 


\title{
Influence of Human Body on Massive MIMO Indoor Channels
}

\author{
Peng-Fei Cui ${ }^{1,2}$, J. Andrew Zhang ${ }^{1, *}$, Wen-Jun $\mathrm{Lu}^{2}$, Y. Jay Guo ${ }^{1}$, Hong-Bo Zhu ${ }^{2, \dagger}$ \\ ${ }^{1}$ Global Big Data Technologies Centre, University of Technology Sydney, Australia \\ ${ }^{2}$ Jiangsu Key Laboratory of Wireless Communications, Nanjing University of Posts and Telecommunications, China \\ Email: *Andrew.Zhang@uts.edu.au, † † zhb@njupt.edu.cn
}

\begin{abstract}
Massive MIMO can dramatically improve capacity and spectral efficiency. However, it is not very clear whether it can significantly improve the signal blockage problem that exists in single antenna systems. In this paper, we investigate the impact of the human body on indoor massive MIMO channels, using practically measured channel data for a 32x8 massive MIMO system in a complex office environment. We introduce a parameter of Power Imbalance (PI) indices to estimate the wide-sense none-stationarity in multiple domains and another parameter of Channel Popularity Indices (CPI) to predict the popularity of MIMO channel. We find that in most cases, the presence of human body still has a non-negligible negative impact. It decreases the ergodic capacity by about $8 \%$ and increases the path loss exponent by 1 . In average, the ergodic capacity for NLOS channels are $15 \%$ higher than that for LOS.
\end{abstract}

\section{INTRODUCTION}

Massive MIMO is becoming an essential technology in mobile and WiFi networks [1], [2]. In recent years, extensive measurement and evaluation activities have been carried out on massive MIMO systems, focusing on three directions. The first is on the properties of outdoor large arrays such as array aperture, decoupling effect, and anti-interference [1], [3]. The second is on distributed multiuser (MU) massive MIMO [4], [5]. The third is on practical problems in using massive MIMO systems, e.g., the spherical wave induced by large-scale arrays, the polarization characteristics and the coupling characteristics within the Rayleigh distance [1], [6], [7].

However, there are limited results reported for indoor massive MIMO channels, and the impact of human body on such channels when the person is using a hand-held device or next to a standalone wireless device. Several important problems for such channels are yet to be investigated. For example, the wide-sense non-stationary or stationary characteristics (or power imbalance) along transmitter (Tx), receiver ( $\mathrm{Rx}$ ) arrays and Tx-Rx domains, pros and cons of multipath on massive MIMO system performance, and the degree of mitigation of human blockage effect by massive MIMO. It is also important to understand the capacity gap between real channels and theoretical ones, particularly for indoor channels where lineof-sight (LOS) and non-LOS (NLOS) propagation may have significant impact on channel correlation across antennas.

In this paper, we investigate the propagation property and the impact of human body on the propagation for a $32 \times 8$ Uniform Planar Array (UPA) MIMO system in an indoor

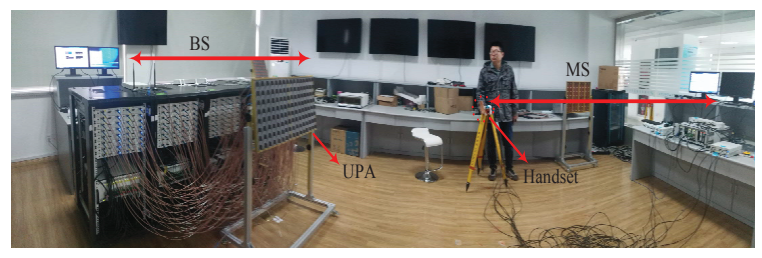

(a) Real office environment and measurement system.

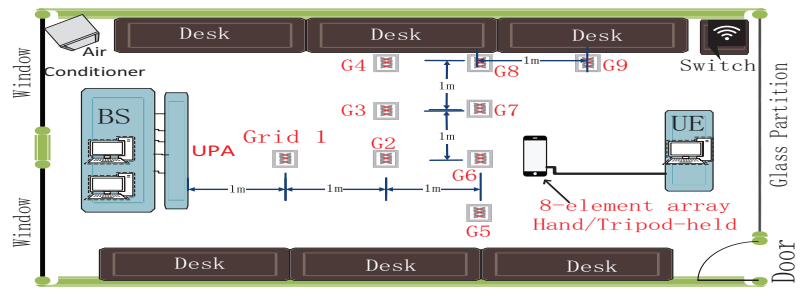

(b) Real office environment and measurement system.

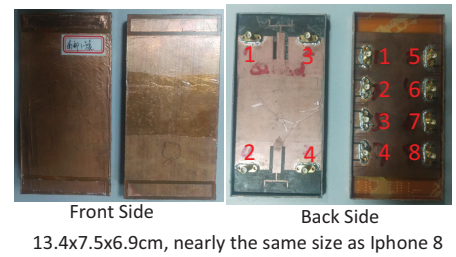

(c) Handset Antenna array

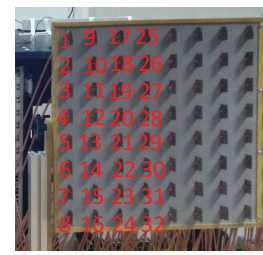

(d) BS antenna array

Fig. 1: The illusion of (a) Real office environment and measurement system (b) MS Antenna array (c) BS antenna array and Indices.

environment with measurements obtained in multiple indoor LOS/NLOS, Body/NoBody scenarios. We first introduce the measurement setup in Section II. We then propose a method in Section III for evaluating the power imbalance and channel popularity in LOS/NLOS, Body/NoBody cases. In Section IV, we analyze the capacity, correlation and angular power spectrum for channels in multiple scenarios. 

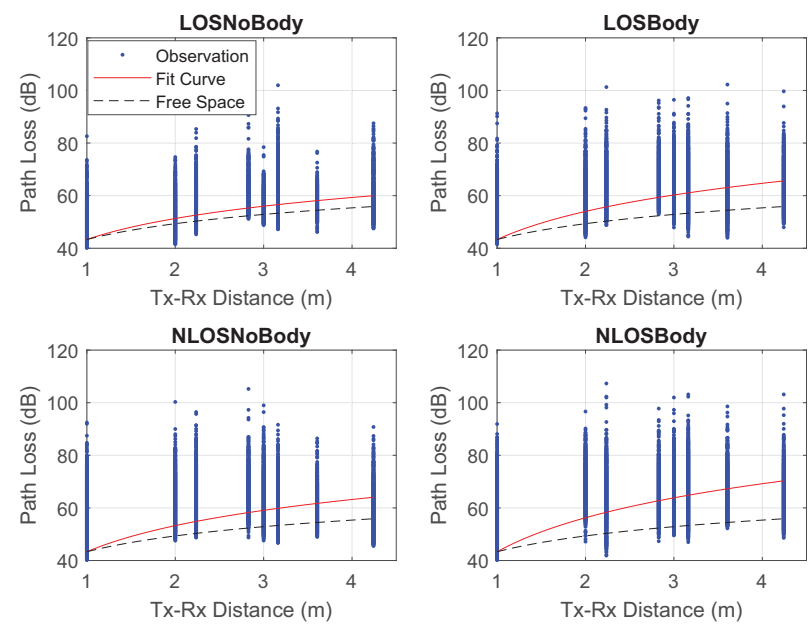

Fig. 2: Path loss curves for 4 typical scenarios with path loss values averaged over Tx and Rx array.

\section{Measurement Setup}

Our measurement campaign was conducted in a typical office environment as shown in Fig. 1 (a) and (b). A large UPA which contains 128 antenna elements is used to emulate the Base Station (BS) and an 8-element UPA is used to emulate the Mobile Station (MS), as shown in Fig. 1 (c) and (d). During the measurement, only 32 Radio Frequency (RF) chains are active, as shown in Fig. 1 (d). The Synchronous Massive MIMO Measurement sounding system (S3M) [2], [8] is used to generate 1200 -subcarrier signals with $0 \mathrm{dBm}$ transmitting power in each transmitting port. The central frequency is 3.5 $\mathrm{GHz}$ with $20 \mathrm{MHz}$ bandwidth and the sampling rate is 30.72 MHz.

The centre of transmitting and receiving arrays are kept at the same height during the measurement. Nine grids across the office are chosen to deploy the 8-element handset array with the transmitter-receiver $(\mathrm{Tx}-\mathrm{Rx})$ distances ranging from 1 to 5 meters. In order to assess the impact of human body on MIMO channels in an indoor environment, at each grid we did measurements for four scenarios: line-of-sight (LOS) connection with a handset fixed on a tripod, LOS with someone holding the handset in a calling gesture, nonLOS (NLOS) with the handset placed on a tripod, and NLOS with the handset held by a person. We denote the four scenarios as LOS_NoBody, LOS_Body, NLOS_NoBody and NLOS_Body, respectively. The S3M system synchronously records 300 snapshots for each Tx-Rx link at each grid. Each snapshot contains 12 complex data from subcarriers evenly distributed over $20 \mathrm{MHz}$ bandwidth. Thus, we obtain a 5dimensional datasets $\mathbf{H}_{t, f, d, m, n}^{C a s e}$ for each scenario, with the data amount of 8294400 complex values $(300 \times 12 \times 9 \times 32 \times 8)$. In $\mathbf{H}_{t, f, d, m, n}^{\text {Case }}$, the parameters $t, f, d, m, n$ represent the indexes of snapshots, subcarriers, grid points, receive and transmit antennas, respectively. More details about the measurement campaigns and S3M system can be referenced to [9], [10].
TABLE I: Path Loss Parameters for 4 typical scenes $\left(P L_{0}=\right.$ $43.3 \mathrm{~dB})$

\begin{tabular}{cccc}
\hline Cases & PLE & shadow $\delta_{X}$ & RMSE \\
\hline LOS NoBody & 2.7 & 4.6 & 5.3 \\
LOS Body & 3.6 & 5.8 & 7.5 \\
NLOS NoBody & 3.3 & 5.7 & 9.2 \\
NLOS Body & 4.3 & 6.3 & 9.5 \\
\hline
\end{tabular}

\section{Channel ImbalanCE ChaRACTERIStiCS}

\section{A. Path Loss Models}

In order to evaluate the effect of adjacent human body on path loss and signal coverage, we adopt the widely used logdistance path loss formula to model all measured signals at different grids as

$$
\overline{P L(d)}=P L_{0}+10 * k * \log _{10}(d)+\overline{\delta_{X}}
$$

where $P L_{0}$ is the free-space path loss at 1 meter $(43.3 \mathrm{~dB}$ in our work), $k$ is the Path Loss Exponents (PLE) and $\overline{\delta_{X}}$ is the mean-zero Gaussian shadowing variable averaging across Tx and Rx UPA domains.

Note that (1) can only predict the average power across Tx-Rx arrays. The specific power prediction for each TxRx antenna pairwise $P L(d, m, n)$ is dependent on the power imbalance in Tx and Rx array and other domains. We introduce the concept of array shadow factor $\delta_{X}(m, n, d)$ to characterize this effect. Due to its wide non-stationary property [3], it is tricky to evaluate this parameter.

Table I shows the parameters for all scenarios obtained using curve fitting technologies. It can be observed that the path loss is affected by both the LOS/NLOS condition and human proximity. Path loss model for the LOS_NoBody case has the minimum PLE and shadow values while NLOS_Body has the maximum values. Human proximity in LOS is equivalent to NLOS state except that the RMSE in NLOS case is obviously larger. Compared with the typical value of 3 for standard shadow deviation and 5 for RMSE of the conventional singlelink channels, the key parameters for the measured massive MIMO channels here are slightly larger. The main reason is that massive scatters for large aperture system is about $1-8.5 \mathrm{~m}$ in this work and within the Fresnel zone (Rayleigh distance) according to

$$
0.62 * \sqrt{D^{3} / \lambda}<d_{\text {Fresnel }} \leq 2 * D^{2} / \lambda
$$

where $D$ and $\lambda$ are the antenna aperture and wavelength of central working frequency. This means we can not view the tested massive MIMO channels as wide sense stationary over multi dimensions.

\section{B. Signal Level Distributions}

Fig. 3 depicts the cumulative probability distributions of signal levels under four scenarios using the same randomly chosen Tx-Rx antenna pairwise at the grid 6 (G6). Only channels in the Los_Nobody case best follows the Rice distribution while the others follow Log-normal distributions. It seems that under the LOS cases, "UE" held by human evidently reduces 


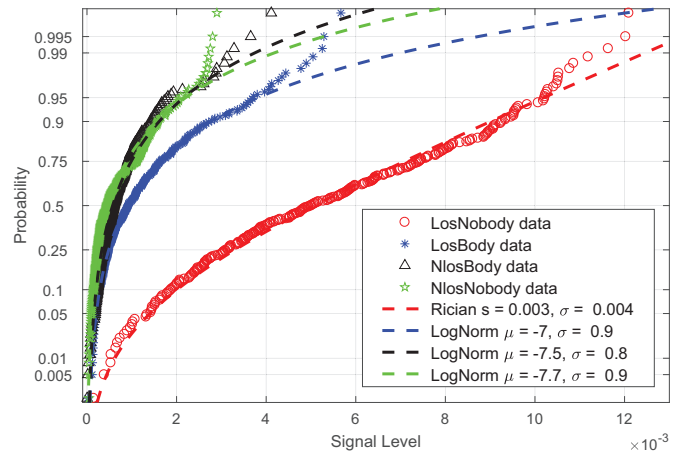

Fig. 3: Cumulative probability distributions of signal levels under four scenarios using the same random chosen $\mathrm{Tx}-\mathrm{Rx}$ antenna pairwise at the same grid 2 .
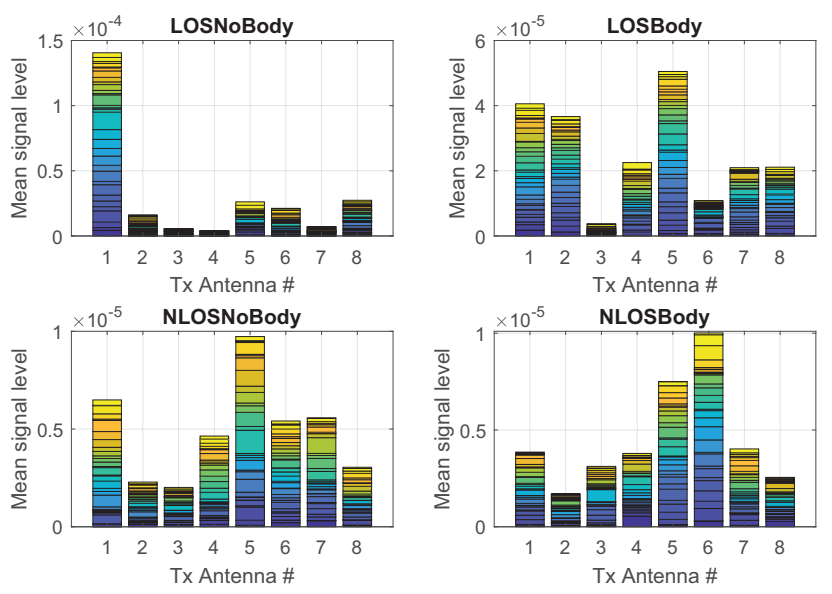

Fig. 4: Stacked received signal strength across Tx antenna array.

the mean signal level to $1 / 5$ of the Nobody case. This causes that distributions having larger tails such as Log-normal and Weibull fit the signal levels of massive MIMO channels under real environments better (LOS_NoBody is just too idealistic in practice).

\section{Signal Level Variation among Tx/Rx Antenna Array}

To explore the impact of adjacent human on none-wide sense stationary features across $\mathrm{Tx}$ arrays, we average the signal levels across temporal, frequency and spatial dimensions and stack each received signal according to the transmitting antenna number shown in Fig. 4. Antenna 1 in LOS_NoBody contributes over $70 \%$ effective transmitting power. Human proximity in the LOS case greatly reduces such imbalance while in NLOS there is no such function.

\section{Power Imbalance among Different Domains}

To quantitatively analyze such power imbalance over different domains, we calculate the standard variation along
TABLE II: Power imbalance values of four scenarios on five different dimensions (Units are all $\mathrm{dB}$, except for SVS.)

\begin{tabular}{cccccc}
\hline Dimensions & LosNB & LosBody & NLosNB & NLosBody & Mean \\
\hline Rx Antenna & 5.6 & 6.5 & 4.0 & 4.6 & 5.0 \\
Tx Antenna & 4.1 & 4.9 & 3.5 & 3.6 & 4.0 \\
SVS(Tx-Rx) & 13.5 & 14.0 & 12.0 & 12.9 & 13.1 \\
Time & 6.1 & 8.1 & 5.5 & 6.4 & 6.5 \\
Frequency & 7.4 & 4.1 & 5.3 & 6.1 & 5.7 \\
\hline
\end{tabular}

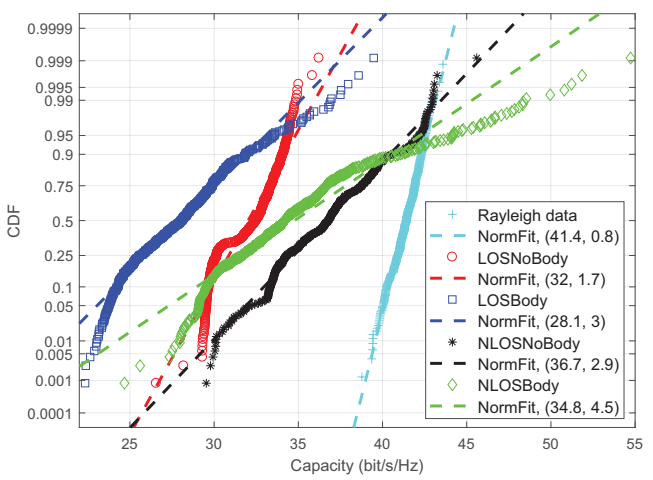

Fig. 5: Distributions of capacity at 6 th measurement grid.

specific domain to obtain its power imbalance. Take the power imbalance in the temporal domain as an example,

$$
\begin{gathered}
\mathrm{PI}_{\text {Time }}=\boldsymbol{s t d}_{\text {Time }}\left(\frac{1}{F D M N} \sum_{f} \sum_{d} \sum_{m} \sum_{n}\right. \\
\left.20 * \log _{10}(\|H(t, f, d, m, n)\|)\right),
\end{gathered}
$$

where $\boldsymbol{s t d}_{\text {Time }}$ means to obtain the standard variation along the time domain, and $F, D, M, N$ are the total numbers of subcarriers, measured grid points, receive and transmit antennas, respecitively. Define the Singular Value Spread (SVS) of channel transfer matrix $\mathbf{H}_{t, f, d}(m, n)$ as the condition number of this matrix, i.e. the ratio between the maximum and minimum singular value of the matrix $\lambda_{\max } / \lambda_{\min }$. Since the correlation between Tx and Rx domains has a great impact on channel stability and channel performance, we use SVS as the power imbalance indicator for Tx-Rx (Transceiver) domain.

Table II depicts the power variations across 5 dimensions. Interestingly, imbalances in the NLOS cases are nearly all less than those in LOS cases. This means that unlike conventional channels, the massive MIMO channels in LOS in indoor environment are more stable and popular. The imbalance increases in the order of NLOS_NoBody, NLOS_Body, LOS_NoBody and NLOS_Body, which corresponds to the order of descending performance as will be shown later.

\section{CAPACity And Factors Affecting it}

\section{A. Capacity VS Number of Antennas}

There exists large capacity gaps between i.i.d Rayleigh and channels in four measurement cases. Fig. 8 shows that channels in all cases best follow the Normal distributions while their ergodic capacity varies. When the number is 8 , in relation to the capacity for Rayleigh, the capacity for NLOS_NoBody 


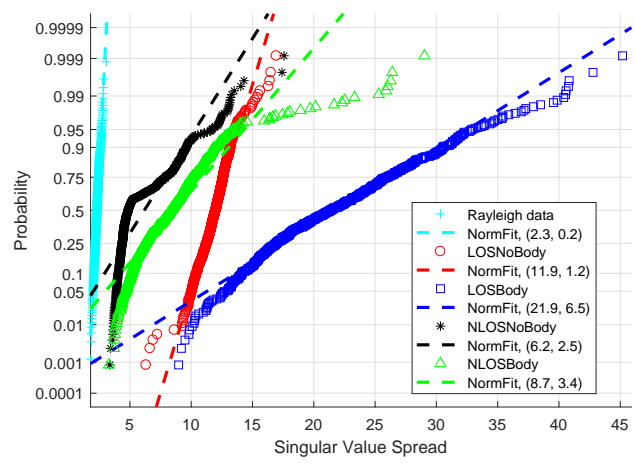

Fig. 6: Distributions of Singular Value Spread (SVS) for all scenarios at the 6th grid.

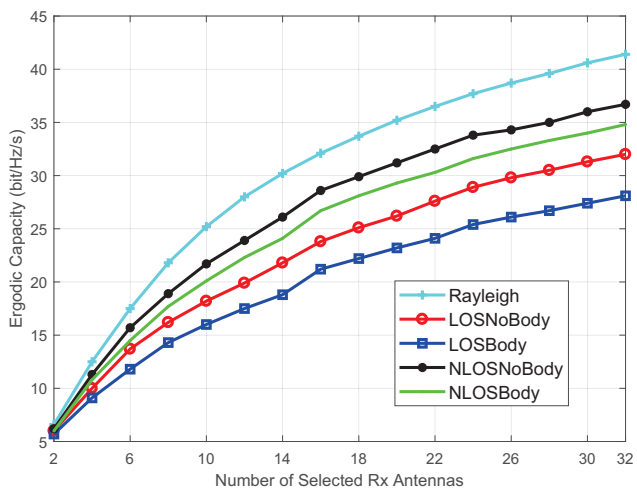

Fig. 7: Capacity variation with numbers of $\mathrm{Rx}$ antennas for all scenarios, $N_{T x}=8, S N R=10 \mathrm{~dB}$.

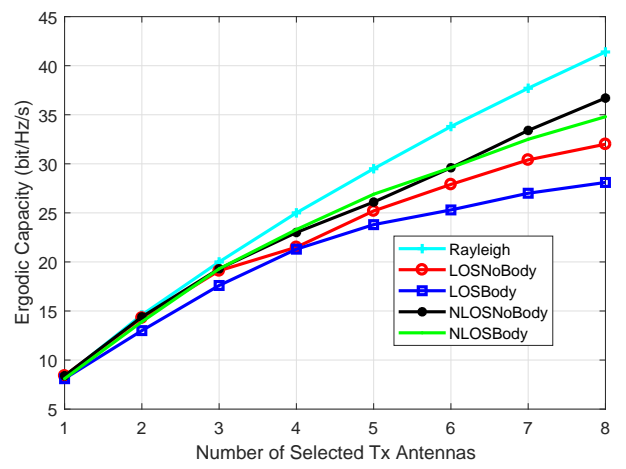

Fig. 8: Capacity variation with number of Tx antennas for all scenarios, $M_{T x}=32, S N R=10 \mathrm{~dB}$.

(36.7 bps $/ \mathrm{Hz}$ ) is $90 \%$, and in the worst case, capacity for LOS_Body $(28.1 \mathrm{bps} / \mathrm{Hz})$ is only $69 \%$. Human proximity causes capacity loss of about $12 \%$ in LOS and 5\% in NLOS cases. Comparatively, the SVS shown in Fig. 6 shows the opposite trends.

Fig. 7 and Fig. 8 depict the ergodic capacity for 4 cases when randomly choosing $\mathrm{Rx}$ and $\mathrm{Tx}$ numbers separately. Ergodic capacity approximately linearly increases with the number of $\mathrm{Rx} / \mathrm{Tx}$ antenna increasing. It is found that the ratio between the capacity for the "worst" LOS_Body cases and Rayleigh almost remains as $69 \%$ with the number of
TABLE III: Parameters for empirical capacity formula and CPI

\begin{tabular}{ccccc}
\hline Cases & $\mathrm{a}$ & $\mathrm{b}$ & $\mathrm{CPI}$ & $\frac{C(\text { case }, 32,8)}{C(\text { Rayl }, 32,8)}$ \\
\hline i.i.d Rayleigh & 7.6 & 0.74 & 1 & 1 \\
LOS NoBody & 5.8 & 0.63 & 0.80 & $77 \%$ \\
LOS Body & 5.1 & 0.57 & 0.72 & $72 \%$ \\
NLOS NoBody & 6.8 & 0.67 & 0.90 & $90 \%$ \\
NLOS Body & 6.3 & 0.66 & 0.86 & $86 \%$ \\
\hline
\end{tabular}

BS antennas increasing, while it decreases rapidly with the number of UE antennas increasing. This means that the human adjacent effect is negligible for mobile devices with multiple antennas.

\section{B. Channel Popularity Indices}

The sub-linearly increasing rate of capacity for measured channels is important for designing and evaluating massive MIMO systems. We propose an empirical capacity prediction method and a parameter of Channel Popularity Indices (CPI) for MIMO system design based on least square curve fitting. The empirical capacities, which are obtained via fitting the curves of Shannon capacity, are given by

$$
\left\{\begin{array}{l}
C_{R x}(\text { case }, m)=a(\text { case }) * m^{\frac{1}{2}} \\
C_{T x}(\text { case }, n)=9 * n^{b} \\
C_{T x, R x}(\text { case }, m, n)=\sqrt{C_{R x}(\text { case }, m) * C_{T x}(\text { case }, n)} \\
=\sqrt{9 a * m^{\frac{1}{2}} n^{b}}
\end{array}\right.
$$

where $C_{R x}($ case,$m), C_{T x}($ case,$n)$ and $C_{T x, R}($ case, $m, n)$ represent the capacity as a function of the antenna numbers of the Rx, Tx and Rx-Tx pairwise for a given channel scenari$\mathrm{o} / \mathrm{case}$, respectively. The coefficients of the power functions can be changed according to the scenario. The CPI is expressed as,

$$
C P I=\sqrt{\frac{a(\text { case })}{a(\text { Rayl })} * \frac{b(\text { case })}{b(\text { Rayl })}}
$$

where $a($ case $)$ and $b($ case $)$ are the feature parameters for specific measurement case, $a(R a y l)$ and $b(R a y l)$ are parameters of i.i.d Rayleigh MIMO channels. Table III shows the fitted parameters for the empirical capacity prediction and CPI. An example for the measurement at grid 6 is also provided. Both the CPI and empirical capacity formula are very simple but works well. It is useful for estimating the performance of practical large-scale MIMO communications.

\section{Correlation Characteristics}

The inter-channel correlation of indoor massive MIMO channels along 5 domains is found to be very high. For example, the mean correlation over the Rx array domain is between 0.35 and 0.8 , and it is $0.77,0.72,0.55$ and 0.74 for the other four domains. The individual correlation varies at different measurement grids.

Fig. 9 shows a typical Rx inter-channel correlation map at grid 2. The Rx correlations for NLOS cases are much smaller than those for LOS, which is consistent with the widely known acknowledged fact. The correlations within 8 antennas in each 

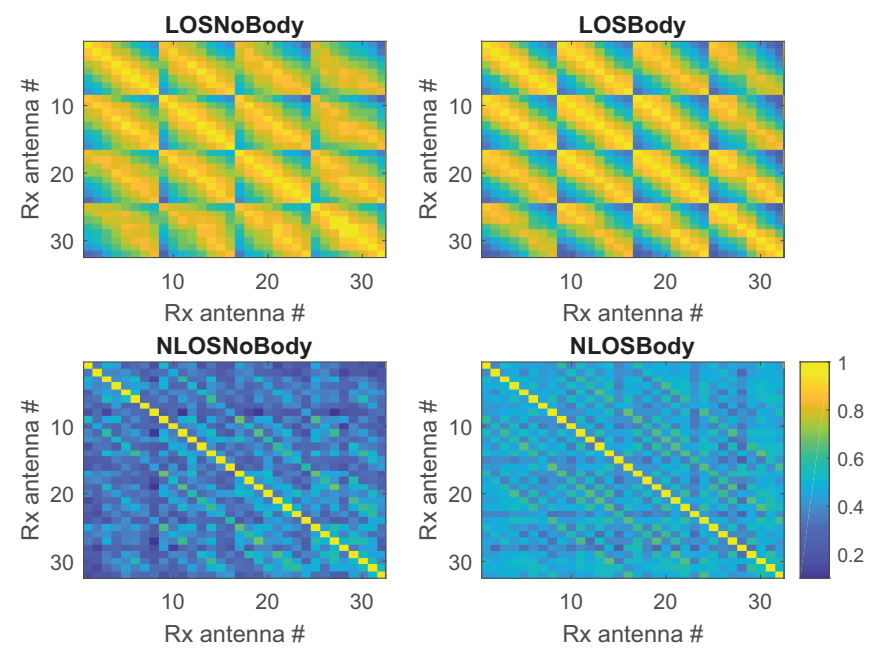

Fig. 9: Typical inter-channel correlation map across receiving array on measurement grid 2 for all scenarios. Mean correlation $0.73,0.69,0.37,0.46$.
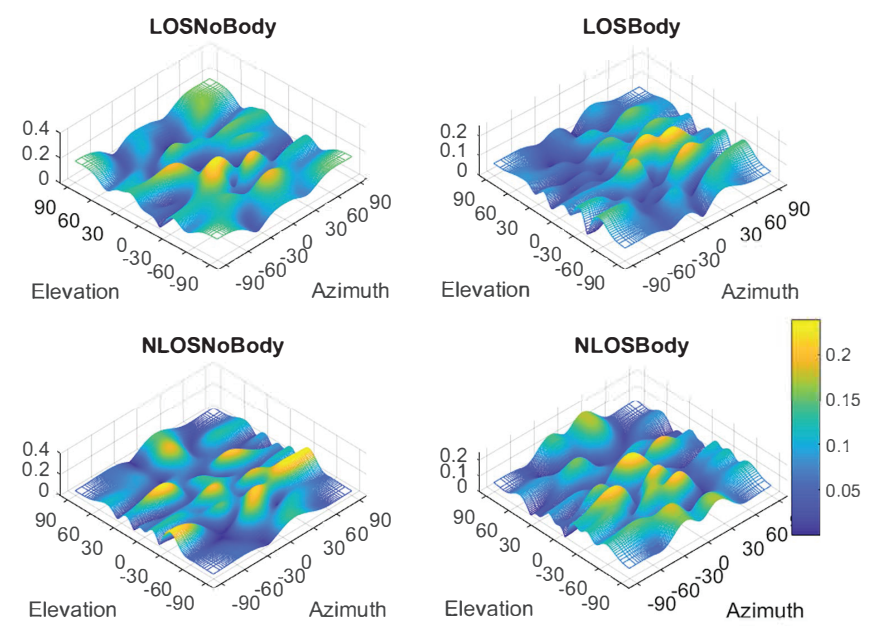

Fig. 10: Arriving angular power spectrum across received antenna array for randomly chosen snapshot, subcarrier and measurement location.

column are larger than those across columns. The correlations within each row in the Rx array are also significantly larger than the inter-row correlations. This makes the correlation map present multiple symmetric and parallel diagonal peaks ribbons forming similar "salmon streaks".

\section{Angular Power Spectrum}

Angular power analysis is the basis for 3D MIMO channel modeling. By using the DFT based angular analysis method in both elevation and azimuth directions, the angular profiles are obtained for the measurements over snapshots, grid points and subcarriers for four cases, as shown in Fig. 10. We find that the number of significant clusters in two NLOS scenarios are generally twice as many as those in LOS. Meanwhile, the presence of adjacent humans decreases the amplitude of significant clusters nearly by half. The significant clusters which are widely observed are another important reason why the expected capacity and anti-fading ability in NLOS scenarios are superior to those in LOS.

\section{CONCLUSiON}

This paper presents experimental studying results for the impact of human body on massive MIMO indoor channels. It is found that human body has a great influence on both LOS and NLOS channels. It can increase the path loss exponents by 1 and reduce the ergodic capacity by $12 \%$ in LOS and $5 \%$ in NLOS cases. In LOS cases, such degradations are mainly due to the large power loss, and no significant improvement is observed in the correlation across $\mathrm{Rx}$ array and angular power maps. In NLOS cases, such degradations can partially be explained by the increasing power imbalance in multiple dimensions, the reduced $\mathrm{Rx}$ correlation and the attenuation of significant clusters caused by human body. Therefore, the influence of human body is significant in indoor massive MIMO channels and shall be considered in massive MIMO system design.

\section{ACKNOWLEDGEMENT}

This work is partially funded by National Nature Science Foundation Council (NSFC) with grant numbers 61427801 and 61871233. It is also supported in part by Jiangsu Scientific Innovation Research of University Graduate with grant number KYLX16 0657. The authors would like to thank Dr. Yu Yu, Jun She, etc. for their assistances in the measurement campaigns.

\section{REFERENCES}

[1] F. Rusek, D. Persson, B. K. Lau, E. G. Larsson, T. L. Marzetta, O. Edfors, and F. Tufvesson, "Scaling up mimo: Opportunities and challenges with very large arrays," IEEE signal processing magazine, vol. 30, no. 1, pp. 40-60, 2013.

[2] E. Luther, "5g massive mimo testbed: From theory to reality," white paper, available online: https://studylib. net/doc/18730180/5g-massivemimo-testbed-from-theory-to-reality, 2014.

[3] S. Payami and F. Tufvesson, "Channel measurements and analysis for very large array systems at $2.6 \mathrm{ghz}$," in Antennas and Propagation (EUCAP), European Conference on. IEEE, 2012, pp. 433-437.

[4] X. Gao, O. Edfors, F. Rusek, and F. Tufvesson, "Massive mimo performance evaluation based on measured propagation data."

[5] P. Harris, S. Zang, A. Nix, M. Beach, S. Armour, and A. Doufexi, "A distributed massive mimo testbed to assess real-world performance and feasibility," in Vehicular Technology Conference (VTC Spring), 2015 IEEE 81st. IEEE, 2015, pp. 1-2.

[6] S. Jaeckel, L. Raschkowski, K. Börner, and L. Thiele, "Quadriga: A 3-d multi-cell channel model with time evolution for enabling virtual field trials," IEEE Transactions on Antennas and Propagation, vol. 62, no. 6, pp. 3242-3256, 2014.

[7] Z. Zhou, X. Gao, J. Fang, and Z. Chen, "Spherical wave channel and analysis for large linear array in los conditions," in Globecom Workshops (GC Wkshps), 2015 IEEE. IEEE, 2015, pp. 1-6.

[8] J. Vieira et al., "A flexible 100-antenna testbed for massive mimo," in Globecom Workshops (GC Wkshps), 2014. IEEE, 2014, pp. 287-293.

[9] Y. Yu, P.-F. Cui, J. She, Y. Liu, X. Yang, W.-J. Lu, S. Jin, and H.-B. $\mathrm{Zhu}$, "Measurement and empirical modeling of massive mimo channel matrix in real indoor environment," in Wireless Communications \& Signal Processing (WCSP), International Conference on. IEEE, 2016, pp. $1-5$.

[10] J. She, C. Gao, Y. Yu, P.-F. Cui, W.-J. Lu, S. Jin, and H.-B. Zhu, "Measurements of massive mimo channel in real environment with 8antenna handset," in Wireless Communications and Signal Processing (WCSP), International Conference on. IEEE, 2017, pp. 1-4. 